\title{
Sous la peau : les mutations subjectives des personnages de Marie NDiaye
}

\author{
Maïté Snauwaert \\ Campus Saint-Jean, Université d'Alberta
}

De En famille, avec sa protagoniste que personne ne reconnaît, à Autoportrait en vert, avec sa narratrice qui ne reconnaît personne, l'identité est chez Marie NDiaye dysphorique, voire impossible, sujette à la fuite, inapte à se cristalliser de façon définitive. À sa place se dégage une subjectivité mouvante, incertaine, impressionnée par ses rencontres, qui évoque le modèle de sujet nomadique et ouvert à l'altérité proposé par Rosi Braidotti (2011, p.16) Dans son article «Marie NDiaye's Discombobulated Subject», Lydie Moudileno relève quant à elle : "what Dominique Viart calls "the subject in disarray" - the condition of a contemporary subject ill-equipped to interpret a 
reality whose logic always seems to escape her ${ }^{1} »$ (p. 84). Ce désarroi, cette désintégration apparents du sujet ne conduisent pas à un résultat unilatéral qui serait celui d'une victimisation même si nombre des protagonistes de NDiaye sont, à un moment ou à un autre, abusés, exploités, humiliés ou ridiculisés, souvent dans leur corps. Plutôt, ces personnages font preuve d'une capacité sans fin d'adaptation, de réagencement, accompli sinon au niveau moléculaire, du moins à travers les changements de peau et les modifications de couleur, les blessures et autres affections de leur chair, jusqu'à l'épuisement et parfois jusqu'après leur mort. Cette transformation incessante se fait au gré de ce que requièrent les situations, comme si l'on assistait à un processus d'évolution qui, au lieu de s'étaler sur plusieurs millénaires, prenait forme dans l'instant. En ce sens, ces figures ambiguës s'inscrivent dans le nomadisme posthumain que Rosi Braidotti lit, dans la lignée de Georges Canguilhem et de Donna Haraway, comme «the French tradition of bodily materialism that argues [...] that any theory of subjectivity worthy of its name must take into account the embodied and organic structure of the subject ${ }^{2}$ " (2006, p. 197). L'ethos mis de l'avant par ces figures de la métamorphose est celui d'une précarité et d'une vulnérabilité telles que Judith Butler les a examinées dans le cadre d'une critique de la guerre (2009) et telles que l'éthique du care propose d'en faire les éléments définitoires de l'humain

\footnotetext{
1 «Le sujet décontenancé de Marie NDiaye»: «ce que Dominique Viart appelle "le sujet en proie au désarroi" - la condition d'un sujet contemporain mal équipé pour interpréter une réalité dont la logique semble toujours lui échapper. » (Ma traduction)

2 « la tradition française du matérialisme physique qui soutient [...] que toute théorie de la subjectivité digne de ce nom doit prendre en compte la structure organique et incarnée du sujet » (Ma traduction).
} 
(Molinier et al., 2009). Cette subjectivité mouvante n'est ainsi ni bonne, ni mauvaise; mais elle met en cause toute vision d'un sujet unifié, solide et limité, bien qu'elle ne sacrifie pas, paradoxalement, la notion d'une intégrité intérieure qui transcenderait ou préexisterait aux états indéfiniment changeants du corps.

Ainsi caractérisé par les métamorphoses, les transpositions mais aussi le pouvoir d'affirmation, ce modèle de sujet, dont le corps est sans cesse soumis aux effets de son environnement, est porté à son paroxysme par l'éloquent Trois femmes puissantes. Dans ce roman, lauréat du Prix Goncourt 2009, les protagonistes féminins - et parfois masculins apparaissent comme d'abord faibles et fuyants, aux prises avec des formes de délabrement. Celles-ci pourtant n'empêchent pas une forme de puissance, au sens où la conçoit Gilles Deleuze dans sa lecture de Spinoza (1981/2003) : non en tant que pouvoir mais en tant que capacité d'effectuation. Ainsi, quelles que soient les situations auxquelles ils doivent faire face, les personnages continuent de se transformer pour les affronter ou, plus souvent, les endurer, au prix d'une patience minutieuse qui leur devient une sorte de dignité, quelque chose d'intérieur du moins et d'à soi à quoi malgré tout tenir. Du sentiment intérieur des protagonistes émerge ainsi ce qu'on pourrait appeler une dignité sans morale, apparentée à une endurance dont la vertu de continuation devient elle-même une morale, comme dans la lecture que propose Philippe Forest des personnages de Faulkner (2010, p. 43-63). C'est cette capacité à continuer qui semble définir une nouvelle puissance chez les protagonistes de la romancière, sans cesse affectés bien plutôt qu'agissants, même si des actions au sens narratif se produisent aussi dans le roman. La troisième des protagonistes du roman, Khady Demba, héroïne 
d'une sorte de fable de l'émigration, porte à son paroxysme cette puissance infinie de mutation. Mais avant d'en venir à elle, j'aimerais proposer un bref détour par une autre fable, publiée quant à elle dans un contexte non romanesque.

\section{Arbitraire de l'identité}

La brève fable qui ouvre, comme en exergue, l'essai de Pap Ndiaye, frère de Marie NDiaye, sur La Condition noire, jette une lumière rétrospective sur l'œuvre de celle-ci. Intitulée Les Sours, elle vient mettre en scène, dans une narration extérieure, deux sœurs métisses dont l'une est presque blanche tandis que l'autre est très noire. La première, traitée comme une sorte de miraculée, jouit des considérations réservées aux Blancs. Elle développe néanmoins un complexe lié à la condition «noire » qu'elle s'attribue, alors que tous semblent la gommer pour elle, et qui lui fait percevoir toutes les portes comme fermées. Elle a en quelque sorte "intériorisé »sa couleur, vécue comme une condition subjective d'infériorisation. L'autre sœur, à l'inverse, se comporte comme si elle n'était pas noire, comme si rien ne l'entravait ni ne la distinguait, et cela, ajouté à ses compétences laissées libres de ce fait de se développer, lui vaut une entière réussite sociale. Cependant, cette conquête se paye également d'un prix individuel, d'une stratégie de déni longuement entretenue qui n'est pas sans évoquer la Fanny d'En famille, dont la couleur de peau n'est jamais explicitée et dont l'« insoutenable singularité semblait lui être inconnue» (Ndiaye, 1990/2007, p. 300). La fable des deux sœurs montre qu'il n'y a pas d'allégeance 
naturelle ou de sentiment d'appartenance inné à une race, celleci relevant d'abord d'une construction sociale, souvent fantasmatique, comme l'a bien analysé Michael Sheringham pour la pièce Papa doit manger (2007, p. 24), y compris pour les sujets concernés.

La fable est éloquente, a fortiori dans le contexte d'un essai de sociologie sous-titré Essai sur une minorité française. Elle illustre le souci de celui-ci de sortir «le fait d'être noir» (titre de la première partie) de tout essentialisme. Le terme de « condition » est choisi par Pap Ndiaye en ce qu'il désigne « une situation sociale qui n'est ni celle d'une classe, d'un État, d'une caste ou d'une communauté, mais d'une minorité, c'est-à-dire d'un groupe de personnes ayant en partage, nolens volens, l'expérience sociale d'être généralement considérées comme noires » (p. 31, je souligne). Loin de définir une essence ou un sentiment intérieur, la « condition noire » est un fait politique et social, à l'historicité variable. Rassemblant les nuances d'un spectre autour d'un terme agglomérateur arbitraire qui a le nom d'une couleur, elle est le résultat d'une médiation et d'une approximation que traduit la modalisation: "généralement considérées comme». Elle est un statut social, une « expérience» faite au contact des autres, apposée, voire projetée de l'extérieur, sans correspondance interne. Elle ne dit rien de l'identité et de la coïncidence qu'elle suppose entre sentiment de soi et reconnaissance par les autres. Dans « condition», il est alors possible d'entendre un conditionnel : je (ne) suis noir-e (que) si quelqu'un dit que je le suis. En redoublant la formule : «l'enquête a été facilitée par le fait que je sois considéré comme noir» (p.27), l'auteur insiste sur l'inexistence d'un état de nature. Il y va d'une affirmation, qui peut être faite (ou non, comme le montre la nouvelle) par le 
« porteur » de la couleur, ou, comme c'est le cas le plus souvent, par ses observateurs, et l'essai vise à « éclaire[r] la variété des identités choisies des personnes noires » (p. 31, je souligne). La couleur unique cède la place au pluriel et à la diversité des choix individuels. Ces choix, ne correspondant pas nécessairement aux attendus sociaux, peuvent s'accompagner d'une infinité de degrés aux résonances politiques. Dans ce contexte, la «norme chromatique » dont il est question au chapitre sur le « colorisme », "c'est-à-dire sur les distinctions et hiérarchies sociales qui existent depuis l'esclavage entre les Noirs selon leur degré de mélanine » (p. 31), fait ressortir la littéralité de la notion de couleur, à la fois sa contingence (seul élément issu de la «nature ») et son arbitrarité (les connotations sociales dont elle s'accompagne).

Une telle problématisation incite à reconsidérer le régime de couleurs propre aux univers de Marie NDiaye. Dans l'Autoportrait marqué de cette teinte, par exemple, le vert des femmes en vert est la couleur d'un désagrément projeté par la narratrice sur les êtres de son entourage ou les rencontres subites qui la mettent mal à l'aise. On ne saurait dire alors si le vert émane de celles-ci, de la narratrice, ou de la chimie fausse de leur rencontre ${ }^{3}$. La couleur joue alors le rôle d'un substitut dans une approche impressionniste du monde, qui, parce qu'elle nous fait prendre en grippe certaines couleurs, mène au racisme. Si le degré auquel un individu se perçoit comme noir et celui auquel il l'est par des tiers ne se rencontrent jamais, parce que des valeurs drastiquement différentes y sont associées, alors cette "condition » ressortit à la palette des couleurs comme celle des goûts, éminemment subjective. Dans Rosie

\footnotetext{
${ }^{3}$ J'ai proposé une étude (2009) plus approfondie de cette ambiguïté.
} 
Carpe, Andrew Asibong relève le caractère agressif de la couleur blanche, qui surgit sous diverses formes pour minimiser l'humanité de la rosâtre Rosie :

White [...] is presented in NDiaye's prose as not just a mere detail. Instead, it is seen as a kind of supreme, unadulterated whiteness gone berserk in its aggressive solidity, ready to melt and liquefy all the lesser, more fragile, vulnerable or somehow "impure" manifestations of humanity in the novel - of which the pink (but also oddly yellow) Rosie figure herself will, paradoxically, be the prime example ${ }^{4}$. (p. 118)

La couleur peut ainsi aussi bien être un trait du monde environnant, ou bien s'y diffuser et le contaminer. Elle n'est en tout cas jamais anodine, souvent hostile, comme pour souligner la fragilité et, comme l'écrit Asibong, la vulnérabilité des protagonistes humains.

\section{La couleur de la peau}

Tout se passe, dans les romans de Ndiaye, comme si chacun avait en puissance une couleur toujours prête à sortir, à trahir le sujet par le jeu de quelque métissage subit ou de la chimie d'une rencontre, conduisant à la honte d'être révélé. Car la honte domine dans ces récits de couleurs craintes qui hantent comme des ancêtres; récits de nature seconde susceptible à

\footnotetext{
${ }^{4}$ Le blanc n'est pas présenté comme un simple détail dans la prose de NDiaye. $\mathrm{Au}$ contraire, il est vu comme une sorte de blancheur suprême et pure devenue folle furieuse dans son agressive solidité, prête à fondre et à liquéfier dans le roman toutes les manifestations d'humanité moindres, plus fragiles, vulnérables ou d'une certaine façon "impures" - dont la rose (mais aussi étrangement jaune) Rosie elle-même sera, paradoxalement, l'exemple parfait. (Ma traduction)
} 
tout moment de livrer malgré le sujet une couleur en laquelle il ne se reconnaît pas. C'est ce que le psychanalyste Jacques Roisin nomme dans ses travaux sur le traumatisme psychique «la honte de la déshumanisation »:

Cette honte surgit face au spectacle de l'extrémité à laquelle peut être réduit l'être humain [...] elle est causée par la répulsion que son état inspire au regard du jugement éthique qui se prononce sur la frontière séparant l'humanité de la nonhumanité: la victime est honteuse d'incarner l'abjection humaine et d'en offrir le spectacle au regard du témoin ou du bourreau, au regard de l'humanité. (p. 83-84)

Le motif véritable de la crise d'identité de Fanny dans En famille, ce que Dominique Rabaté identifie à propos du personnage éponyme de Hilda comme « une sorte de folie de l'identité, dans laquelle le signe n'est plus assuré de renvoyer à un référent stable» (p. 49), est en effet moins lié à sa couleur qu'à l'échec de sa reconnaissance par son milieu familial, qui persiste à l'appeler du faux nom de Fanny. « Chez Marie NDiaye, remarque Rabaté, le lien familial se caractérise par sa nature duplice : donnée irrécusable, il est en même temps absolument gratuit, sans obligation, sauf pour le héros. » (p. 27) La nouvelle « Fanny » doit alors se plier à cette identité, dont elle ignore ce qu'elle recouvre mais qui vient nommer sa honte même : «si d'évidence la honte exige toujours une image de rabaissement, source du sentiment pénible, celle-ci peut surgir selon des causalités de nature diverse jusqu'à ne provenir, dans certains cas, que de la seule dépréciation lue dans le regard d'autrui » (Roisin, p. 83-84). Cette honte semble toutefois chez NDiaye toujours déjà inscrite au creux du personnage, et celui-ci n'a pas d'autre choix que de s'y accrocher, sous peine, sinon, de perdre toute consistance ou toute identité. Roisin ajoute que si « [t]oute honte est liée à l'image dévalorisante reflétée au sujet 
dans le lien social » (p. 85), «l'identification à la déchéance » de la victime "participe du mécanisme d'identification de survie, au sens ou l'attachement à cette identification permet d'éviter le maintien de l'anéantissement» (p. 84). Le psychanalyste insiste ainsi sur «la part active du sujet dans la production de sa honte », à travers la façon dont il se regarde lui-même (p. 85).

La couleur est ainsi une donnée contingente chez NDiaye, une pellicule extérieure sans relation de nécessité avec les protagonistes, bien qu'elle les détermine lourdement, en proportion de leur besoin d'interagir et de se sentir appartenir à une famille, qui pourrait souvent être étendue à la famille humaine. Bien qu'elle ne soit pas une émanation de leur intériorité, elle devient une interface puissante charriant l'imaginaire de leur entourage et le vecteur d'une «honte partagée » dans laquelle tacitement, "victime et témoin », mais peut-être aussi bourreau, «se rencontrent» (Roisin, p. 84). C'est à ce titre qu'elle affecte en retour les sujets qui la portent comme un masque, selon tout l'arbitraire d'une "condition » non choisie. Claire Ducournau note au sujet de Rosie Carpe :

L'opposition du noir et du blanc apparaît donc dans sa dimension arbitraire, socialement construite. Elle ne sert pas, en tout cas, à comprendre les personnages de l'intérieur : les désignations qu'elle suscite épaississent au contraire leur mystère, soit qu'elles se retournent de manière brutale, de l'éloge au blâme, soit qu'elles soient l'objet de jugements de valeur tout extérieurs. (p. 106-107)

De plus, la critique fait remarquer que le noir et le blanc ne sont pas, au plan chromatique, "des couleurs au sens strict du terme [...]. Ce sont des non-couleurs de peau, des catégories de jugement spontanées et creuses, qui épousent un regard extérieur souvent hostile, en laissant les subjectivités dans l'opacité. » 
(p. 107) Ce qui la conduit à demander : «De quelle couleur sont donc réellement les personnages?» (p.107) Dominique Rabaté parle ainsi d'« une sorte de fantastique quasi ethnologique» de NDiaye (2008, p. 37). Car si la question raciale est centrale dans l'œuvre, à tout instant son centre aveugle, elle se manifeste en tant que question de la couleur, qui en rend l'arbitrarité en la détachant précisément du politique, la ramenant au domaine plus subjectif et irrationnel des goûts et des couleurs. Elle vise moins une ethnicité particulière que le jeu de fantasmes par lequel les «degrés de mélanine » se voient attribuer des valeurs, faisant ressortir le fait que ces attributions opèrent sur la base d'éléments tellement superficiels que même leur dimension physiquement tangible fait problème en fonction des perceptions des uns et des autres, créant le clivage entre regards intérieur et extérieur. Dans l'univers de NDiaye, la couleur de la peau est tout au plus un résultat, en aucun cas une origine ou une source culturelle. Pourtant, et c'est là toute son ambiguïté pour les sujets concernés, elle se vit comme un jugement social intériorisé, et son déni - pris pour une décision de liberté - aboutit le plus souvent à son renforcement, son intensification sous la forme de la honte.

Non seulement la couleur de la peau ne coïncide pas avec le sentiment de soi, elle fausse celui-ci et conduit à séparer l'identité réelle de celle attendue socialement, au point de rendre la première incertaine ou douteuse : vaguement honteuse, occasion d'une grande solitude puisqu'elle ne se voit pas validée par le groupe humain. L'identité alors n'est plus le gage d'un être "identique à soi-même», comme dans la définition première de Ricœur (qu'il jugeait insuffisante pour rendre compte de l'identité humaine), mais un label donné de l'extérieur, comme dans le ministère de l'Immigration, de l'intégration, de l'identité nationale 
et du développement solidaire qui fut la création fugitive de Nicolas Sarkozy 5 .

Dans les romans de Marie NDiaye, c'est la nécessité supposée du lien familial - ou parfois des liens amicaux ou communautaires, donc empreints de social - qui ne cesse ainsi d'être relativisée à travers la couleur de peau, présentée comme aléatoire jusque dans ses attaches culturelles et le "patrimoine d'origine» dont elle serait porteuse. Sollicitée, comme le rapporte Andrew Asibong, pour faire partie d'une étude sur les romancières africaines, Marie NDiaye répond qu'elle n'est née ni n'a grandi en Afrique, qu'elle n'en connaît pour ainsi dire rien, n'a pratiquement pas connu son père. Avec son flegme coutumier, elle précise qu'elle souhaitait signaler cela au solliciteur, «ne sachant si vous étudiez des romancières aussi superficiellement africaines que je le suis » (p. 111, je souligne). D'Afrique comme de son père elle n'a que la couleur, de sorte qu'elle est en quelque sorte faussement noire, et toute l'œuvre travaille à revenir sur la contingence stupéfiante d'un tel lien, cette "manière d'être d'une réalité (être ou chose) susceptible de ne pas être ${ }^{6} »$.

\section{Intégrité et humanité}

Dans son excellente étude "Travel Sickness », Andrew Asibong cite en épigraphe cette phrase de Cioran : «Dans tout citoyen d'aujourd'hui gît un métèque futur. » Il est difficile d'imaginer

\footnotetext{
${ }^{5}$ On se souvient que Marie NDiaye a décidé de quitter la France lorsque l'ancien ministre de l'Intérieur est devenu Président de la République.

6 Article "contingence», Trésor de la langue française informatisé, http://atilf.atilf.fr/.
} 
une déclaration plus à même de décrire le processus de mutation, et de déchéance apparente, des personnages de Marie NDiaye, exemplairement et nouvellement incarné par le personnage final de Trois femmes puissantes. Or si l'identité des personnages est en fuite, si «[l]es héros de Marie NDiaye ne peuvent plus avoir de destin, seulement des noms qu'ils s'efforcent de conserver, de remplir d'un sens qui les fuit » (Rabaté, 2003, p.54), et si leur corps même fuit par ses blessures, ses métamorphoses, ses obligations à muter (à changer de forme, de couleur), c'est aussi qu'on les chasse d'un ordre humain d'abord défini comme ordre social : «Elle avait ignoré quelle forme prendrait leur volonté de se débarrasser d'elle mais, que le jour viendrait où on lui ordonnerait de s'en aller, elle l'avait su ou compris ou ressenti (c'est-à-dire que la compréhension silencieuse et les sentiments jamais dévoilés avaient fondé peu à peu savoir et certitude) dès les premiers mois de son installation dans la famille de son mari, après la mort de celui-ci. » (p. 247)

Pourtant, lorsqu'elle est libérée de l'emprise de sa bellefamille, Khady retrouve la sensation claire de son corps jeune et puissant : « elle prit conscience du travail de ses muscles [...], de leur vigueur, de leur indéfectible présence qu'elle avait oubliée, de tout son jeune corps solide auquel elle ne prêtait plus la moindre attention et dont elle se ressouvenait, qu'elle retrouvait» (p. 263). Parce qu'alors "arrachée à sa hantise», celle d'une grossesse qui a échoué à venir (p. 249), elle redevient "elle-même, celle qu'elle était avant son mariage » (p. 249), celui-ci apparaissant comme une prison sociale malgré la gentillesse du mari. Cette libération individuelle la mène à une incohérence sociale, mais laisse aussi le champ libre à cette retrouvaille du corps, bientôt suivie de celle de la pensée 
comme si l'une et l'autre étaient indissociables : «Qu'elle fût capable de formuler intérieurement de telles questions l'étonnait et la troublait. / Son esprit travaillait, cherchait, souffrait d'être ainsi soumis à la réflexion mais la progression de ce labeur en elle la fascinait et ne lui déplaisait pas. » (p. 276) Rosi Braidotti écrit, à partir des travaux de Michel Foucault, que «one becomes a subject through a set of interdictions and permissions, which inscribe one's subjectivity in a bedrock of power $^{7} »(2011$, p. 12).

Or, tandis qu'elle vivait dans un état d'humiliation proche de la disparition totale, Khady n'a jamais douté de son intégrité, grâce à «l'aïeule qui l'avait élevée et protégée et qui avait su reconnaître, bien qu'elle l'eût traitée avec rudesse, qu'elle était une petite fille particulière nantie de ses propres attributs et non une enfant parmi d'autres»(p.253). Cette intégrité se caractérise par une singularité intrinsèque et irréductible qui, bientôt, se désigne et s'affirme par l'identité onomastique :

De telle sorte qu'elle avait toujours eu conscience d'être unique en tant que personne et, d'une certaine façon indémontrable mais non contestable, qu'on ne pouvait la remplacer, elle Khady Demba, exactement, quand bien même ses parents n'avaient pas voulu d'elle auprès d'eux et sa grand-mère ne l'avait recueillie que par obligation - quand bien même nul être sur terre n'avait besoin ni envie qu'elle fût là. (p. 253-254)

Or son contentement - «[e]lle avait été satisfaite d'être Khady, il n'y avait eu nul interstice dubitatif entre elle et l'implacable réalité du personnage de Khady Demba » (p. 254) provient non de qualités singulières, originales, mais de cette

\footnotetext{
7 «quelqu'un devient sujet à travers une série d'interdictions et de permissions qui installent la subjectivité sur un socle de pouvoir» (Ma traduction).
} 
unicité même, indice à la fois d'une humanité et d'une égalité radicales : "Il lui était même arrivé de se sentir fière d'être Khady car, avait-elle songé souvent avec éblouissement, les enfants dont la vie semblait joyeuse [...], ces enfants-là n'étaient pas plus humains que Khady Demba qui n'avait pourtant, elle, qu'une infime portion de bonne vie.» (p. 254) Ce sentiment de soi qui émane entièrement de l'intérieur, d'une conviction acquise il y a longtemps, voire implantée dès la naissance, se traduit par une foi totale en une forme d'auto-définition : «À présent encore c'était quelque chose dont elle ne doutait pas qu'elle était indivisible et précieuse, et qu'elle ne pouvait être qu'elle-même.» (p. 254) Cette foi est indépendante des situations de vie, qui apparaissent comme de simples occurrences aléatoires à subir, à travers lesquelles patienter : «Elle se sentait seulement fatiguée d'exister et lasse des vexations, même si ces dernières ne lui causaient pas de réelle douleur.» (p.254) Ce sentiment indéfectible de soi, indépendant de la recherche de la vie bonne qui fait l'horizon de la morale, n'est pas sans évoquer la critique de l'aspiration au bonheur des sociétés occidentales telle que la formule Lauren Berlant dans Cruel Optimism (2011).

Car son congédiement de sa belle-famille après la mort de son mari propulse Khady Demba vers une nouvelle connaissance proprioceptive, un éveil progressif de sa conscience qui est pourtant autre chose qu'une réflexion volontaire :

Aussitôt ses pensées prirent congé de la réflexion et [...] elle sentit que son esprit se laissait envahir par la brume familière non plus traversée pourtant des visages morts du mari ou de l'aïeule mais des images qu'attrapaient ses yeux au cours du trajet, dans les rues où l'entraînait cet homme et où elle n'avait 
pas souvenir d'être jamais allée, encore que, se dit-elle soudain, elle avait pu les parcourir dans son état habituel d'hébétude, de prostration mentale, et ne pas se le rappeler - tandis qu'il lui semblait que, ce matin, les plus modestes scènes qui s'égrenaient au long du chemin insistaient délicatement pour se fixer en transparence derrière l'écran de ses songes. (p. 261-262)

Cette métamorphose révèle peu à peu son intégrité d'être humain - «hors d'atteinte, à l'abri de son inaltérable humanité » (p. 314) -, incarnée par la croyance forte en sa propre identité, sans cesse réitérée et couronnée par la certitude onomastique (p. 264, 272, 280, 284, 296, 297, 304, 307 et 310 ), repère certain dans la confusion et le chaos où la laisse un univers dans lequel elle dépend largement des autres. Cependant, au cours de sa marche inopinée à travers la ville, elle redécouvre des endroits qu'elle a déjà parcourus, et quelque chose de sa propre intégrité à travers le temps lui revient, au point qu'on assiste à une exemplification de l'identité ipse conçue par Paul Ricœur, cette identité à soi-même qui persiste malgré les changements vécus dans le temps :

Et elle ressentit alors si pleinement le fait indiscutable que la maigre fillette farouche et valeureuse qui discutait âprement le prix du mulet, et la femme qu'elle était maintenant, qui suivait un étranger vers un rivage semblable, constituaient une seule et même personne au destin cohérent et unique, qu'elle en fut émue, satisfaite, comblée, et que ses yeux la picotèrent, et qu'elle en oublia l'incertitude de sa situation ou plutôt que cette précarité cesse de lui paraître aussi grave rapportée à l'éclat exaltant d'une telle vérité. (p. 264)

À l'opposé du personnage de jeune fille de la Fanny d'En famille, l'identité émane, pour la veuve répudiée par sa bellefamille, de l'intérieur: elle est une souveraineté, un sentiment inaliénable et puissant d'insubstituabilité, que souligne sa régulière réitération onomastique. Si dès l'éponyme Rosie Carpe, 
Dominique Rabaté mettait en garde contre cette fiction de stabilité : «à force de répéter la tautologie d'un nom propre (Rosie Carpe est bien Rosie Carpe), s'insinue le soupçon que cette alliance naturelle n'a rien d'évident, que rien peut-être ne garantit ni le nom ni l'identité » (2003, p. 49), une intégrité s'affirme chez Khady Demba qui semble l'emporter positivement. Alors qu'elle est le jouet des circonstances, suivant un inconnu puis un autre, déplacée d'un endroit à un autre par la nécessité obscure d'une émigration, elle acquiert la conviction intime que sa vie lui appartient: "Aussi, en décidant de l'accompagner, n'ébranla-t-elle nullement sa propre conviction qu'elle dirigeait maintenant elle-même le précaire, l'instable attelage de son existence. / Bien au contraire. » (p. 286)

Il n'est ainsi pas impossible qu'à la déshumanisation par la honte de la Fanny d'En famille s'oppose la réhumanisation ou la «posthumanisation» de Khady Demba, selon la conception de Rosi Braidotti, dont Stefan Herbrechter offre une synthèse éclairante :

Both as a woman and a feminist, Braidotti's allegiance to "man" (humanism's notion of what it means to be human) has always been "negotiable," as she says. This means that the demise of the figure of the human and the advent and insistence of the figure of the posthuman can be welcomed as a chance. The posthuman reminds "us" that we have never been as human as humanism tried to make us believe. The myth of one humanity, based on universal values, an essential human "nature" and human exceptionalism with regard to nonhuman others, has always worked to exclude some humans that didn't correspond to the ideal which tacitly underlies the apparent universalism: there have always been fine gradations within the category of the human, according to gender, race, class, culture, nation, etc. This is why the feminist Braidotti begins her book by saying that "not 
all of us can say, with any degree of certainty, that we have always been human, or that we are only that".8. $(2013$, p. 6)

Fortement fondée par un sentiment de soi qui trouve sa confirmation dans la répétition à l'envi de son nom, cette dignité posthumaine de Khady Demba n'a plus alors besoin d'en passer par les catégories traditionnelles de la reconnaissance. Elle est elle-même à elle-même, peu importe les masques et les marques physiques dont ses voyages vont l'affubler, les rôles sociaux (l'épouse sans enfants, la belle-sœur, la prostituée, la fille) dont la dotent ses rencontres. Versatile, elle semble pouvoir se décomposer et se recomposer au gré des nécessités du moment, chacun de ceux-ci n'étant pas vécu comme historique, mais comme un déplacement d'affect, une altération de l'humeur environnante. Dans sa réflexion sur le posthumain, Rosi Braidotti écrit: "This is why the issue of subjectivity is so central to this book: we need to devise new social, ethical and discursive schemes of subject formation to match the profound transformations we are undergoing. [...] The posthuman condition urges us to think critically and creatively about who

\footnotetext{
${ }^{8}$ À la fois en tant que femme et en tant que féministe, l'allégeance de Braidotti à "l'homme" (la notion humaniste de ce que signifie être humain) a toujours été "négociable", comme elle le dit. Cela signifie que la disparition de la figure de l'humain et l'émergence et l'insistance de la figure du posthumain peuvent être accueillies comme une chance. Le posthumain "nous" rappelle que nous n'avons jamais été aussi humains que l'humanisme a tenté de nous le faire croire. Le mythe d'une seule humanité, fondée sur des valeurs universelles, une "nature" humaine essentielle et un exceptionnalisme humain vis-à-vis d'autres non humains, a toujours travaillé à exclure certains humains qui ne correspondaient pas à l'idéal sous-tendant tacitement l'universalisme apparent: il y a toujours eu de fines gradations au sein de la catégorie de l'humain, en fonction du genre, de la race, de la classe, de la culture, de la nation, etc. C'est pourquoi la féministe Braidotti commence son livre en disant que "nous ne pouvons pas tous dire avec certitude que nous avons toujours été humains, ou que nous sommes seulement cela". (Ma traduction)
} 
and what we are actually in the process of becoming. ${ }^{9} \gg(2013$, p. 12) Si le corps et ses manifestations, les formes de son apparition aux autres sont en constante mutation, malgré la solidité d'un nom propre qui n'appartienne qu'à soi, c'est bien la subjectivité ce processus de devenir. Car, sans qu'on sache qui est elle - son nom ne vaut que pour elle -, Khady Demba continue tout au long de la nouvelle à être soignée et nourrie, à passer littéralement de mains en mains, sans que jamais une identité ne lui soit apposée de l'extérieur.

\section{Mutations et corporéité}

Ainsi le corps de Khady est-il atteint, irrémédiablement déchiré, sans que cela porte atteinte à son intégrité morale. À travers ses déchéances successives, malgré des moments de découragement et la trahison de l'homme auquel elle s'est attachée, sa détermination demeure intacte. Khady devient alors une figure étonnamment deleuzienne : manifestant que, dans son absence totale de pouvoir, elle a encore de la puissance, cette capacité d'un corps d'en affecter d'autres, comme lorsque, se prostituant, elle transmet son infection vaginale à ses clients, agissant en somme sur ceux qui la possèdent (p. 303). Cet état est sans moralité chez Khady; il relève d'une simple affection physique, qui fait porter

\footnotetext{
${ }^{9}$ «C'est pourquoi le problème de la subjectivité est si central dans ce livre : nous avons besoin d'inventer de nouveaux schèmes sociaux, éthiques et discursifs de formation du sujet afin de répondre aux transformations profondes que nous sommes en train de subir. [...] La condition posthumaine nous exhorte à penser de façon critique et créative à qui et ce que nous sommes réellement en train de devenir. » (Ma traduction)
} 
l'attention, ou le doute, sur le caractère construit, évolutif du «naturel»: «Elle considérait maintenant cet épuisement comme la condition naturelle de son organisme. » (p. 310)

Le corps et la pensée sont ainsi des affects, non orientés par un Mal transcendant. C'est un élément très insistant des entretiens de la romancière d'ailleurs, qu'elle refuse d'accorder un statut final bienveillant ou malveillant à la plupart de ses figures (Jacques, 2001-2005). Ces agencements perpétuels peuvent être positifs ou délétères; ils témoignent, comme chez Spinoza, d'états particuliers et transitoires, non-téléologiques. Ils sont toutefois entièrement dépendants de la rencontre, des moments d'influence d'autrui et des possibilités différentes d'y réagir :

Elle se sentit fugacement redevenir faible, tributaire de la détermination et des connaissances d'autrui comme des intentions indécelables qu'on nourrissait à son propos, et la tentation l'effleura, par fatigue de vivre, de se résoudre à cette subordination, de ne plus réfléchir à rien, de laisser de nouveau sa conscience voguer dans le flux laiteux des songes. (p. 291)

L'inertie apparaît ainsi par instants comme un état désirable, ce que Dominique Rabaté a appelé dès Rosie Carpe «l'éternelle tentation de l'hébétude » (2003), mais rien de plus; d'ailleurs, Khady n'y cède pas: «Un peu écœurée, elle se reprit. » (p. 291) Tout un cas est fait du vivant, de la possibilité même de la vitalité, si amoindrie soit-elle, par opposition au mort et au disparu. Songeant à son mari, accablée « de chagrin et de pitié » pour lui, « bien qu'elle eût mal et se donnât encore des tapes sur la poitrine elle ne pouvait s'empêcher de se sentir chanceuse. » (p. 266) Car l'essentiel est la survie - le fait d'être encore là dans le moment prochain - de sorte qu'une sélection 
s'opère parmi les forces utiles pour entretenir ce devenir. Blessée gravement au mollet :

Elle marchait d'un coin à l'autre de la cour en boitillant et s'efforçait de s'habituer et de dresser son corps à cette entrave afin que cette nouvelle situation, le pas ralenti et la douleur continue, devînt une part d'elle-même qu'elle pourrait oublier ou négliger, reléguée parmi les circonstances, comme les histoires pénibles du passé de Lamine qui, ne pouvant servir, risquaient seulement de freiner ou de dévier le développement encore jeune, incertain de ses pensées en y insinuant des éléments de trouble, d'incontrôlable souffrance. (p. 290)

La peau, dans ce contexte, devient partie prenante d'une corporéité déliquescente (" elle tâta son mollet blessé, sentit sous ses doigts du sang, des chairs déchiquetées », p. 283 ; «les deux morceaux de chair étaient nettement séparés», p. 287), voire purulente («la vulve gonflée et douloureuse et le vagin brûlant, irrité », p. 296 ; « le bruit de succion de son dos mouillé sur le matelas tout imbibé de sueur et l'infime clapotement dans sa vulve brûlante », p. 304), au point que Khady en espère la transformation : "Et comme, de surcroît elle éprouvait une grande faim, elle souhaita ardemment d'acquérir bientôt un corps insensible, minéral, sans désirs ni besoins, qui ne fût qu'un outil au service d'une intention dont elle ignorait encore tout mais comprenait qu'elle serait bien forcée d'en trouver la nature. » (p. 286)

Lorsqu'après ces douloureuses mésaventures, Khady Demba, enfin armée de son échelle pour franchir la frontière, se déchire aux barbelés et retombe du côté où elle était venue, cette peau du corps finit littéralement par lâcher : « les barbelés arrachaient la peau de ses mains et de ses pieds et elle pouvait maintenant s'entendre hurler et sentir le sang couler sur ses bras, ses épaules » (p. 316). Or dire que ce côté où elle retombe 
« en arrière » est le mauvais serait erroné, ou à tout le moins incertain : car si Khady Demba suit le vieux rêve que son ancien guide puis traître lui a présenté comme une condition de salut passer la frontière qui permettrait de rejoindre l'Europe (p. 286) -, ce mot, «l'Europe », n'est jamais assorti d'aucune description, et rien ne permet de croire que quelque chose de meilleur l'y attend, hormis le désir partagé par ceux qu'elle rencontre de franchir ce "grillage séparant l'Afrique de l'Europe » (p. 313). Asibong conclut ainsi son article, au sujet d'Autoportrait en vert: " we are reminded of the only thing about her status of which we can be sure: here is a subject who is prey to liquidation, but who carries on to its other side. ${ }^{10}$ » (2009, p. 124) Les notions de bien ou de mal, de mauvais côté, politique, territorial, humain (la proxénète se révèle aussi soignante, le sauveteur se révèle un traître) cèdent du terrain, car c'est la frontière qui fondamentalement déchire : le fait de devoir choisir; d'avoir à passer d'un côté ou de l'autre, tandis que la chute de Khady est paradoxalement douce : « tombant en arrière avec douceur et pensant alors que le propre de Khady Demba, moins qu'un souffle, à peine un mouvement de l'air, était certainement de ne pas toucher terre, de flotter éternelle, inestimable, trop volatile pour s'écraser jamais, dans la clarté aveuglante et glaciale des projecteurs » (p. 316). Une chute par laquelle « son crâne heurt[e] le sol » (p. 316) mais qui permet son envol, renversant la polarité. Comme depuis le début de cette partie du roman, Khady devient, sans qu'il faille envisager un terme à ce processus, ni une valeur ou forme finale. On pourra ainsi considérer ou bien que l'histoire de Khady Demba

\footnotetext{
10 «nous sommes rappelés à la seule chose concernant son statut dont nous pouvons être sûrs : voilà un sujet qui est en proie à la liquidation, mais qui continue de l'autre côté. » (Ma traduction)
} 
se termine dans un épuisement de sa résilience et une critique de celle-ci, humainement caduque si elle n'est plus orientée par un projet, un espoir, mais n'est plus que reconduction à tout prix - voire au prix de sa vie - d'un effort pour repousser ses propres limites, comme paraît le suggérer le modèle néo-libéral. $\mathrm{Ou}$ bien au contraire qu'elle s'est défaite et affranchie des entraves d'une identité rendue non nécessaire, obsolète par un milieu environnant auquel Khady ne peut qu'être assujettie. Si la subjectivité s'entend comme l'ensemble des forces affectives et morales, intellectuelles et physiques qui animent l'humain, celle de Khady est intacte tandis que s'est depuis longtemps dissoute une identité qui ne lui était plus d'aucun secours; voire à laquelle elle est devenue inversement proportionnelle.

\section{Étrange réalisme}

Le fantastique de Marie NDiaye, oscillant entre des ordres différents de réalité, provoque une réflexion sur les limites de l'humain. La mise en cause de la réalité tangible du corps; ses fluctuations de sujet à objet; d'humain à animal; de vivant à revenant; le défaut de coïncidence entre sa perception intérieure et extérieure; la concurrence enfin entre identité et subjectivité et la remise en cause de leur synonymie dans le canon occidental, façonnent le texte étrange de Trois femmes puissantes. Dans ces variations subies plutôt qu'agies, ou simplement éprouvées par les personnages, ce qui frappe est la difficulté de leur attribuer une valeur définitive, euphorique ou dysphorique. Dominique Rabaté relève ainsi que " [l'] extrême singularité » des récits de NDiaye « ne se réduit jamais à ce qui 
ne saurait en constituer la morale ou le sens politique, car ce que marque justement le fantastique, c'est l'impossibilité à subsumer sous une catégorie unifiante le fonctionnement de la réalité » (p.37). Si l'indignation sociale, politique ou humanitaire se propose souvent pour le lecteur, surtout devant la dernière de ces femmes puissantes, abandonnée, prostituée, infectée, déplacée, il devient pourtant délicat de donner un label moral aux états du corps. Khady Demba elle-même, « immergée tout entière dans la réalité d'un présent atroce », se contente de se représenter cette période comme «transitoire, persuadée que ce temps de souffrance aurait une fin et qu'elle n'en serait certainement pas récompensée (elle ne pouvait penser qu'on lui devait quoi que ce fût pour avoir souffert) mais qu'elle passerait simplement à autre chose qu'elle ignorait encore mais qu'elle avait la curiosité de connaître » (p. 297). Tout au plus les états du corps sont-ils, comme chez Deleuze, bons ou mauvais : permettant plus ou moins de puissance, sans qu'on puisse affirmer si le résultat obtenu est un échec ou une réussite, une aliénation ou une libération, ces critères n'ayant plus cours. Lorsqu'elle songe à son mari mort, à sa grand-mère qui l'a aimée, «ce n'étaient là que pensées et non regrets car aussi bien elle ne déplorait pas son état présent, ne désirait à celui-ci substituer nul autre et se trouvait même d'une certaine façon ravie, non de souffrir mais de sa seule condition d'être humain traversant aussi bravement que possible des périls de toute nature » (p. 312). Ou si ces critères ont encore cours, qui sont ceux d'un régime économique néo-libéral, le roman les dépeint en creux; les personnages les subissent sans y avoir accès, car les rênes en sont tenues ailleurs. Mais par là ils accèdent aussi à une certaine indépendance. 
Dans cette réalité dans laquelle les personnages sont empêtrés, qui semble de la consistance pâteuse des rêves ou plutôt des cauchemars, l'univers romanesque met l'accent sur la capacité de réinvention indéfinie qui est attendue d'eux. Au final, l'inconstance, une forme d'indéfinition semblent grever le genre humain - ou peut-être le libérer? -, dans l'écho des derniers textes de Samuel Beckett. Une forme d'absurde règne sur ces univers (différents dans chaque partie), mais ce n'est en quelque sorte que l'absurde habituel de la condition humaine, qui tout en ignorant les cartes qui lui sont données, n'a pourtant pas le choix de composer avec elles. Or c'est le cadre de référence de la philosophie occidentale, avec son accent fort placé sur la fin et la finalité de la vie humaine, qui semble évacué. Ce modèle "distensionnel» fait place à un modèle «transitionnel», selon les termes du philosophe François Jullien : faisant de la vie entière un processus, "dont chaque moment se découvre et compte à part entière, et est gros du suivant » (p. 68). Dans ce nouvel ordre ou ce nouveau chaos, les individus sont seuls face à des règles qui les dépassent même s'ils les pressentent. Cette oppression ne les rend pas meilleurs, ni plus mauvais. Ils ont seulement, selon un rythme qui apparaît intensifié, à évoluer de présent en présent, sans que la fin soit un but, puisque même elle n'apparaît plus comme un terme sûr ou définitif.

\section{Plasticité et métamorphose}

La déchéance partout présente dans l'œuvre de NDiaye, non sans évoquer l'autre spécialiste en diminutions physiques 
Samuel Beckett, n'est pas nécessairement synonyme d'une perte de puissance. Lydie Moudileno parle d'un sujet déconcerté, décontenancé, "discombobulated», au point que le terme mérite en français d'être pris littéralement. Les corps sociaux, chez Ndiaye, perdent toute contenance; les corps physiques à leur tour toute capacité à être des contenants : ils coulent, fuient de toutes parts (leur incontinence est un trait récurrent; parfois, c'est la matière même de leur chair qui se défait, comme chez Rosie Carpe ou Fanny), mais aussi démangent, torturent (Rudy a des hémorroïdes), en un mot s'humilient: apparaissent comme inférieurs, méprisables, par des paroles ou des actes interprétés comme abaissant leur dignité11. Si cette humiliation est causée par leur propre corps qui leur fait soudain défaut, ne leur sert plus de support - ce que les personnages seraient à même de tolérer, si seulement ils étaient seuls -, elle est surtout relayée par le regard des autres, et c'est là qu'intervient la vraie peine, car c'est là que survient la honte.

Cependant, chez les derniers protagonistes de NDiaye, cette humiliation semble être surmontée tant ils semblent la reprendre à leur compte, s'en faire une forme d'arrogance de ceux qui n'ont plus rien à perdre mais savent tirer parti du peu qu'ils sont. Du moins, elle paraît dissociée de la honte, comme si elle n'était que l'effet - le moment de passage - d'un processus d'adaptation. Alors que, dans les textes précédents, un état ne semblait jamais pouvoir être final, une sorte de point d'orgue est apportée ici, signalée par le «contrepoint» ponctuant chacune des trois histoires. Ce contrepoint joue le rôle de

\footnotetext{
11 Selon la définition du verbe «Humilier» dans le Trésor de la langue française (http://atilf.atilf.fr/).
} 
désuniciser le point de vue, de rappeler que même la souveraineté a des témoins, ou peut-être ici, des bénéficiaires. Moments d'apaisement, ces points d'orgue nous donnent à voir celui ou celle sur qui agit la métamorphose finale - en oiseau dans le cas de Khady, transformation qui fait écho à celle de la première des femmes puissantes, Norah. Dans son Ontologie de l'accident. Essai sur la plasticité destructrice, Catherine Malabou écrit :

Dans l'imaginaire occidental, force est de le remarquer, la métamorphose est rarement présentée comme une réelle et totale déviation de l'être. Elle ne l'est peut-être même jamais. Quelles que soient ses bizarreries [...], les formes qu'elle crée, le résultat des transmutations des infortunés qui en sont les victimes, restent, si l'on peut dire, dans l'ordre des choses. En effet, ce n'est que la forme extérieure de l'être qui change, jamais sa nature. L'être demeure ce qu'il est au sein du changement même. Le présupposé substantialiste est le compagnon de route de la métamorphose occidentale. La forme se transforme, la substance demeure. (p. 15)

Chez NDiaye, ce sont le sentiment intérieur de soi et le nom pour le confirmer qui persistent à travers ou malgré la métamorphose, sans pourtant qu'on puisse dire que la transmutation opérée reste "dans l'ordre des choses », d'abord parce qu'elle est incertaine, allusive ou floue :

C'est moi, Khady Demba, songeait-elle encore à l'instant où son crâne heurta le sol et où, les yeux grands ouverts, elle voyait planer lentement par-dessus le grillage un oiseau aux longues ailes grises - c'est moi, Khady Demba, songea-t-elle dans l'éblouissement de cette révélation, sachant qu'elle était cet oiseau et que l'oiseau le savait. (p. 316)

L'ambiguïté de l'affirmation redoublée - «c'est moi, Khady Demba » - articulant la phrase fait fonctionner en miroir ce nouveau savoir, cette « révélation » qui, si prise en son sens 
religieux, a quelque chose de transcendant, en tant que dévoilement d'une connaissance autrement inaccessible à la raison. Khady songe-t-elle qu'elle est elle-même, Khady Demba, comme elle l'a scandé tout au long de la nouvelle? Ou bien songe-t-elle qu'elle est l'oiseau, ce qui s'apparenterait davantage à une révélation survenant au moment de la mort ? Quoi qu'il en soit, dans ce passage de subjectivité d'une forme physique à une autre (réversible chez Norah et son père), d'un état humain minoritaire à un « devenir-animal » (à prendre ici littéralement) qui serait le gage d'une nouvelle puissance, un croisement se fait entre le monde occulte et le naturel qui n'est pas sans évoquer le vaudou, lui-même issu de la déportation d'esclaves d'Afrique aux Caraïbes, et mêlant sorcellerie et rituels religieux. De sorte que s'il y a un (nouvel) ordre des choses, il réside dans cette concomitance des êtres et des milieux, des règnes animal et humain, du ciel et de la terre, du vivant et du mort, de la grâce et de la malédiction; à même, dans un flux continu, de s'échanger leurs places.

En finale, la fable deleuzienne de Marie NDiaye semble affirmer une fin du pouvoir - incarné par les organes traditionnels de la famille et de l'État - tel qu'il s'exerce, chez Spinoza, sur des quantités entières. À ce pouvoir se substituent des puissances plurielles, s'affirmant selon des degrés et des affects localisés pour échapper aux rêts unifiants du pouvoir. $\mathrm{Au}$ pouvoir de l'identité, donné de l'extérieur (famille, nationalité, regard social) est substitué un devenir intrinsèque des corps, dont les souffrances répétées témoignent d'un état de crise, d'une mutation tantôt lente, tantôt subite. Ce devenir, incarné dans un mouvement continuel évoquant une conception nomadique du sujet, insiste sur le caractère non-euphorique de ce nomadisme, qui ne fait qu'errer d'étrangeté en 
" étrangèreté », au gré d'exclusions que finit par reconduire le sujet lui-même : « lorsque Lamine lui eût fait part de sa propre intention [...], qu'il arriverait un jour en Europe ou mourrait et qu'il n'y avait aucune autre solution au problème qu'était sa vie, il parut évident à Khady qu'il ne faisait là que rendre explicite son dessein à elle.» (p. 286) Le «cruel optimism» de Berlant surgit à nouveau dans cette relativisation tragique de l'immigration vers les pays riches comme chemin contemporain vers une «vie bonne» pour les populations du Sud : «A relation of cruel optimism exists, écrit Berlant, when something you desire is actually an obstacle to your flourishing. ${ }^{12} »(\mathrm{p} .1$ ) Comme dans la philosophie de Rosi Braidotti, le résultat est une fiction politique qui œuvre à contrer, par des modèles et croisements inédits, toute forme d'hégémonie de la pensée qui ferait du «retour du sujet» en Occident un bien universellement partageable.

\section{Point de fuite}

Dans les textes de Marie NDiaye couve la possibilité d'une forme étrangère, proche ou lointaine, qui tantôt glisse, animale, dans la vision périphérique; tantôt, couleur hostile, revêt les formes rencontrées (Autoportrait en vert). Le fantomatique se glisse dans les lieux habités, sur le bord de la Garonne ou à Berlin ( $Y$ penser sans cesse). Des personnes que l'on pensait bien connaître reviennent, mais méconnaissables, porteuses d'une

\footnotetext{
12 «Une relation de cruel optimisme existe, écrit Berlant, quand quelque chose que vous désirez est en réalité un obstacle à votre épanouissement. » (Ma traduction)
} 
identité ou d'une personnalité nouvelles (Autoportrait); ou réclament des lieux qu'elles ont quittés depuis longtemps, mais dont l'habitation implique une substitution d'identité (Rien d'humain).

$\mathrm{Si}$, comme le formulait Michel Serres, «Dieu est notre pudeur», l'animalité est peut-être le dernier refuge lorsque toutes nos forces sociales, politiques, affectives ont été réduites à néant, que nous sommes ramenés à notre seule force vitale. Celle-ci n'est peut-être pas alors un abaissement, mais une retrouvaille, comme chez Khady Demba, avec un corps premier et dernier qui est, à travers ses métamorphoses, lui-même le lieu et l'exercice d'une subjectivité. Cette contre-subjectivité, pourrait-on dire, est un devenir-minoritaire au sens fort, qui se glisse entre les mailles du politique pour devenir politique à son tour, en mode subalterne, sous-jacent, et fait peut-être moins fi qu'il n'y paraît du refuge de cette « inaltérable humanité » dont s'enthousiasme Khady Demba (p. 314). Dans l'introduction de son livre sur le posthumain, Rosi Braidotti écrit: "I take the posthuman predicament as an opportunity to empower the pursuit of alternative schemes of thought, knowledge and selfrepresentation. ${ }^{13}$ » $(2013$, p. 12) Dans ces univers romanesques qui semblent faits de la pâte des rêves, lourds, difficiles à déplacer, faussement «tranquilles » selon un adjectif récurrent des romans, l'inertie de la protagoniste (Rosie), mais aussi sa persistance (Fanny) ou sa volonté aveugle (Khady Demba), jamais découragée malgré les signes que lui envoie l'extérieur,

\footnotetext{
13 «Je prends la situation délicate du posthumain comme une opportunité de rendre possible la poursuite de schèmes alternatifs de pensée, de connaissance et d'auto-représentation. » (Ma traduction)
} 
rencontrent l'hostilité d'entourages d'autant plus menaçants qu'ils sont censés être proches. Les rencontres des personnages avec des oiseaux, que parfois ils deviennent ou que parfois ils écrasent (Rudy à la fin de la deuxième histoire) paraissent alors constituer une forme de nouvel espoir, de nouvel « envol ». Car après tout, s'il faut se transformer jusqu'à être un chien pour survivre, n'ira-t-on pas jusqu'à se transformer en chien? Quel jugement moral pourrait être tiré de là, et par qui ? Ce deveniranimal ici littéralement incarné n'est-il pas la démonstration simplement que, quoi qu'il arrive, nous saurons nous transformer, jusqu'à l'extrême, jusqu'à la dissolution, jusqu'à la liquéfaction s'il le faut? N'est-ce pas d'ailleurs la grande demande des sociétés néo-libérales que cette transformation incessante des ressources humaines? Dominique Rabaté écrit à propos du réalisme magique de NDiaye: "Le programme individualiste du roman occidental touche ainsi à une de ses limites, source de comique et d'effroi.» (2003, p. 54) L'animalité pourrait pointer vers le refoulé d'un monde prétendument civilisé qui apporte pourtant chaque jour de nouvelles preuves de sa barbarie. Ou, jugement de valeur en moins, vers une futurisation de l'humain indéfiniment capable de se modifier au gré des pressions imposées sur lui. Ou encore, représenter une critique de cette infinie et indéfinie mutation, qui ne se joue sur la base d'aucune décision morale, d'aucune constitution politique collective. L'animalisation des personnages de Marie NDiaye pourrait être lue comme une critique du modèle libéral du sujet occidental, nomade contemporain supposé capable d'aller et de vivre partout alors que cela n'est possible que pour une infime partie de la population, politiquement et financièrement privilégiée. 
La fin duelle du roman - l'échec politique et social de Khady Demba d'une part, qui n'a pu franchir la frontière de barbelés; et la réussite de sa transfiguration d'autre part, qui lui permet d'échapper aux limites de son corps - nous laisse avec l'impossibilité d'une réponse simple, et le refus d'en donner une. Si Khady Demba est une héroïne, elle n'est pas une sainte, mais atteint le ciel sous la forme d'un oiseau, poursuivant son existence terrestre pour devenir plus libre mais aussi, il n'y a aucun moyen de le savoir, peut-être plus méchante. Dans le " contrepoint», Lamine, cependant qu' « un oiseau dispara[ît] au loin », rend grâce à «la fille » après avoir rêvé qu' « elle le protégeait ou, au contraire, le vouait au pire» (p. 316). Rabaté écrit : «Être un héros chez Marie NDiaye, c'est toujours être en proie à cette panique pratique, à cette peur devant un monde trop violent [...] dont il s'agit moins de comprendre les règles (elles n'existent pas) que de s'y adapter.» (2003, p.51) Ces « personnages pragmatiques », comme les appelle l'auteur, sont ainsi « ceux qui savent s'adapter à la loi du devenir, qui savent que l'être est une qualité transitoire et inutilement encombrante » (2003, p. 52).

La présente lecture, elle-même en mutation, a tenté de mettre au jour, dans le roman, un refus de toute dialectique réconciliante, des binarismes et des systèmes qui nous obligent à choisir entre deux (blanc ou noir, homme ou femme, victime ou dominateur), parce que nous échangeons nos places constamment, fragiles et forts, incertains et fiers, conscients et aveugles. Les mutations qui s'exercent sur les personnages, jamais entièrement justifiées par la diégèse ou du moins par un système de sens réaliste - vieillissement, rajeunissement, éclaircissement, assombrissement, blessures, et jusqu'à la métamorphose animale - apparaissent comme des 
extrapolations de la façon dont nous changeons à tout instant, en fonction des rôles que nous jouons - parents de nos enfants, enfants de nos parents, femmes désirables dans les yeux de nos pères; ces rôles que les univers romanesques de NDiaye accentuent, accumulent et croisent de façon théâtrale et troublante. Trois femmes puissantes met en évidence que l'être humain n'est pas un être de frontières, avec des bords précis, des contours nets, mais une puissance en mutation. Aussi, lorsqu'un paradigme philosophique, politique, racial, social, spirituel ou genré binarise le monde, sans doute faut-il l'animaliser, le vaudouiser, pour le complexifier, pour ne pas priver le sujet de la conscience multipliée de sa vie.

\section{Bibliographie}

AsIBONG, Andrew. (2009), "Travel sickness: Marie NDiaye, Hervé Guibert and the liquidation of the White FantasySubject », International Journal of Francophone Studies, vol. 12, no 1 , p. 109-125.

BERlant, Lauren. (2011), Cruel Optimism, Duke University Press.

Braidotti, Rosi. (2013), The Posthuman, Cambridge, Polity Press.

- (2011), Nomadic Subjects: Embodiment and Sexual Difference in Contemporary Feminist Theory (2nd Edition), New York, Columbia University Press.

-. (2006), «Posthuman, All Too Human. Towards a New 
Process Ontology », Theory, Culture \& Society, vol. 23, no 7-8, p. 197-208.

ButLER, Judith. (2009), Frames of War. When Is Life Grievable?, New York, Verso Books.

Deleuze, Gilles. (1981/2003), Spinoza. Philosophie pratique, Paris, Minuit, coll. « Reprise».

DuCOURNAU, Claire. (2009), « Entre noir et blanc. Le traitement littéraire de la couleur de peau dans Rosie Carpe et Papa doit manger », Revue des Sciences humaines, no 293, p. 101-117.

FOREST, Philippe. (2010), Le Roman infanticide: Dostoïevski, Faulkner, Camus. Essais sur la littérature et le deuil, Nantes, Cécile Defaut.

HERBRECHTER, Stefan. (2013), "The Roar on the Other Side of Silence... or, What's Left of the Humanities? », compte rendu de Rosi Braidotti, «The Posthuman », Culture Machine, avril, p. 1-13, $<$ http://www.culturemachine.net/index.php/cm/issue/view/11>.

JACQUES, Paula. (2008), «Entretiens avec Marie NDiaye, 20012005 », extraits des émissions « Cosmopolitaine » enregistrées et diffusées sur France Inter les 4 novembre 2001 et 13 février 2005, INA/Cultures France, CD inclus dans Dominique Rabaté, Marie Ndiaye, Paris, Textuel, coll. «Auteurs ».

JULLIEN, François. (2009), Les Transformations silencieuses. Chantiers, I, Paris, Grasset.

Malabou, Catherine. (2009), Ontologie de l'accident. Essai sur la plasticité destructrice, Paris, Leo Scheer, coll. « Variations ».

Molinier, Pascale, Sandra LAUGIER et Patricia PAPERMAN. (2009), Qu'est-ce que le care? Paris, Payot \& Rivages. 
MoudiLENO, Lydie. (2006), «Marie Ndiaye's Discombobulated Subject », SubStance, vol. 35, no 3, p. 83-94.

NDIAYE, Marie. (2011), Y penser sans cesse, avec des photographies de Denis Cointe, Talence, L'Arbre vengeur.

-. (2009), Trois femmes puissantes, Paris, Gallimard.

- (2008), «Préface. Les sœurs », dans Pap NdiAYe, La Condition noire. Essai sur une minorité française, Paris, Calmann-Lévy, rééd. « Folio actuel », p. 11-18.

- (2005). Autoportrait en vert, avec des photographies de Julie Ganzin, Paris, Mercure de France, coll. « Traits et portraits ».

—. (2004), Rien d'humain, Besançon, Les Solitaires intempestifs.

—. (1990/2007), En famille, Paris, Minuit, coll. « Double».

NdiAYE, Pap. (2008), La Condition noire. Essai sur une minorité française, Paris, Calmann-Lévy, rééd. coll. « Folio actuel ».

RABATÉ, Dominique. (2008), Marie Ndiaye, Paris, Textuel, coll. «Auteurs ».

—. (2003), "L'éternelle tentation de l'hébétude », L'Atelier du roman, septembre, p. 48-55.

RoIsin, Jacques. (2010), De la survivance à la vie. Essai sur le traumatisme psychique et sa guérison, Paris, Presses universitaires de France.

Sheringham, Michael. (2007), « The Law of Sacrifice: Race and the Family in Marie NDiaye's En famille and Papa doit manger », dans Marie-Claire Barnet et Edward Welch (dir.), Affaires de famille: The Family in Contemporary French Culture and Theory, Amsterdam, Rodopi, coll. « Faux-titre », p. 23-37. 
SNAUWAERT, Maïté. (2009), « La bataille identitaire dans le texte français contemporain : portraits et photographie chez Marie Ndiaye », Portraits biographiques, La Licorne 84, p. 149-165.

\title{
Résumé
}

On réfléchit dans cet article à la façon dont les corporéités mutantes des personnages de Marie NDiaye proposent une subjectivité nouvelle, qui dépasse le modèle unifié occidental au profit d'un devenir opérant par affections successives sans valeur morale. En particulier, on s'attarde sur la dernière figure de Trois femmes puissantes, Khady Demba, pour la lire comme l'héroïne d'une fable deleuzienne.

\begin{abstract}
This article examines the bodily mutations of Marie NDiaye's protagonists to suggest that they shape a new kind of subjectivity, one that forgoes the rational and unified model of Western philosophy in favor of a becoming with no moral value attached. A particular attention is given to the last character of Trois femmes puissantes, Khady Demba, in order to read her as the heroin of a deleuzian fable.
\end{abstract}

